\title{
Condensation in stochastic mass transport models: beyond the zero-range process
}

\author{
M. R. Evans and B. Waclaw \\ SUPA, School of Physics and Astronomy, University of Edinburgh, Mayfield Road, \\ Edinburgh EH9 3JZ, United Kingdom \\ E-mail: mevans@staffmail.ed.ac.uk, bwaclaw@staffmail.ed.ac.uk
}

\begin{abstract}
We consider an extension of the zero-range process to the case where the hop rate depends on the state of both departure and arrival sites. We recover the misanthrope and the target process as special cases for which the probability of the steady state factorizes over sites. We discuss conditions which lead to the condensation of particles and show that although two different hop rates can lead to the same steady state, they do so with sharply contrasting dynamics. The first case resembles the dynamics of the zero-range process, whereas the second case, in which the hop rate increases with the occupation number of both sites, is similar to instantaneous gelation models. This new "explosive" condensation reveals surprisingly rich behaviour, in which the process of condensate's formation goes through a series of collisions between clusters of particles moving through the system at increasing speed. We perform a detailed numerical and analytical study of the dynamics of condensation: we find the speed of the moving clusters, their scattering amplitude, and their growth time. We finally show that the time to reach steady state decreases with the size of the system.
\end{abstract}

PACS numbers: 89.75.Fb, 05.40.-a, 64.60.Ak

Date April 11, 2018 


\section{Introduction}

Condensation is one of the most ubiquitous forms of phase transition experienced in everyday life. Water vapour condenses into a liquid on cold surfaces such as windows or a cold beverage taken out of the fridge. Fog is an aerosol that occurs when temperature drops below the dew point and water vapour condenses into tiny droplets suspended in air. Condensation can also be seen as small "clouds" in low-pressure zones above aircraft wings when flying in damp weather conditions. Condensation is also extensively used in industry for making liquid oxygen and nitrogen, production of liquefied natural gas, or in petroleum distillation.

All these examples describe a transition from a gas to a liquid state of matter. However, in statistical physics, the notion of condensation is more widely used to describe any process in which a finite fraction of some conserved quantity becomes localized in the phase space. So understood, condensation can occur either in real space or in momentum space. In fact, the most prominent example of statisticalphysics condensation - Bose-Einstein condensation - takes place in the momentum space where a finite fraction of all particles present in the system assumes the lowest energy state. Such generalized condensation occurs in many different systems, both in- and out-of-equilibrium: granular clustering, wealth condensation [1], quantum gravity [2], polydisperse hard spheres [3], hub formation in complex networks [4], or even jamming in traffic flow [5, 6].

Perhaps surprisingly, many features of the generalized condensation transitions observed in the systems listed in the previous paragraph, can be captured by a very simple model called the zero-range process (ZRP) [7]. ZRP is a well-studied, nonequilibrium model of particles hopping between sites of a lattice. In the simplest onedimensional asymmetric version of this model, a particle moves from site $i$ to $i+1$ of a one-dimensional periodic lattice with rate $u\left(m_{i}\right)$, where $m_{i}$ is the occupancy of the departure site $i$. As the rates are totally asymmetric a current always flows and detailed balance cannot be satisfied, thus the steady state is non-equilibrium in nature. When the hop rate $u(m)$ decays slowly enough with $m$ and the density of particles is larger than a certain critical density (the exact value of which depends on the function $u(m)$ ), a finite fraction of all particles condenses onto a single, randomly chosen site.

The advantage of ZRP is that its non-equilibrium steady state has a simple factorised form which is amenable to analysis and has furnished our understanding of the condensation transition. This is why the model is often used as an effective description of more complicated systems, for example systems with more microscopic degrees of freedom, where such a complete analytical description is often impossible.

The characteristic feature of the ZRP is that the hop rate depends only on the departure site and not on the destination site. A natural generalisation is to consider a hop rate $u(m, n)$ where $m$ is the occupancy of the departure site $i$ and $n$ is the occupancy of the destination site $i+1$. Such processes are sometimes referred to as migration processes in the mathematical literature [8]. An example of such process is 




Figure 1. Definition of the misanthrope process: a particle hops from site with $m$ to site with $n$ particles with rate $u(m, n)$.

the 'misanthrope process' [9] whose name originally derived from considering a hop rate $u(m, n)$ as an increasing function of $m$ and decreasing function $n$; an effective repulsion between individuals results. The name 'misanthrope process' is now generally used for any hop rate of the form $u(m, n)$. Such a process has been used, for example, to model link rewiring in complex network models [10]. Another example is the target process introduced in Ref. [11]. Here, the rate $u(m, n)=\left(1-\delta_{m, 0}\right) v(n)$, with some function $v(n)>0$, depends on the occupation of the arrival site.

Our aim in this paper is to analyse a more general case of $u(m, n)$. It has been shown that the misanthrope process has a factorised steady state when certain conditions on $u(m, n)$ (expressed below in Eqs. (14) and (15)) are satisfied. Here we provide examples of hop rates when these conditions are satisfied. A particularly interesting case that we discuss is when the hop rate factorizes:

$$
u(m, n)=w(m) v(n) .
$$

We find that for (11) to yield a factorised steady state requires a further condition (25) on the functions $w(m)$ and $v(n)$.

We also analyse the conditions for condensation to occur. As the steady state has a factorised form the conditions on the weights $f(m)$ will be the same as for the zero-range process. However, there is more freedom in the misanthrope dynamical rules with which to realise this asymptotic behaviour. Moreover, the existence of condensation depends not only on the asymptotic behaviour of $u(m, n)$ as it does for the ZRP, but also on $u(m, 0)$ which gives the hop rate to an empty site. In the case of factorized hop rate (1), the condensation criterion turns out to depend on $v(0)$.

We also expand on our previous work [12] and study the dynamics of condensation for two different choices of the factorized hop rate (1). Both forms of $u(m, n)$ lead to the same steady-state probability distribution but contrasting dynamical behaviour. A particularly interesting case, in which $u(m, n)$ increases with both $m$ and $n$, leads to "explosive condensation" where the time to condensation decreases with increasing system size. This is very similar to "instantaneous gelation" known from the theory of coagulation processes, and we discuss similarities and differences between the two models in the last section.

Beyond factorised steady states it is known that pair-factorised states (also referred to as Markov measures) may occur in some stochastic models. A simple example is the 1+1d solid-on-solid model [13, 14, 15] with a "pinning potential", which describes the 


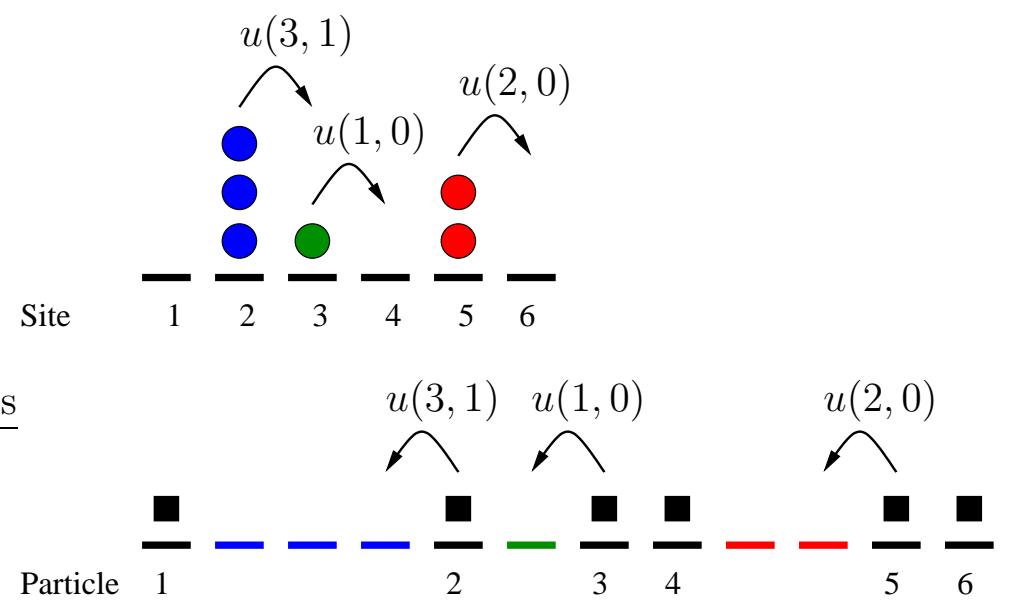

Figure 2. Mapping between the misanthrope process and the total asymmetric simple exclusion process (TASEP).

height of the interface $\left\{m_{i}\right\}$ between two phases and whose microstate probability reads

$$
P\left(m_{1}, \ldots, m_{N}\right)=\exp \left(-J \sum_{i}\left|m_{i}-m_{i+1}\right|+U \sum_{i} \delta_{m_{i}, 0}\right),
$$

with two parameters $J, U$. The $J$-term describes the tendency of the system to form straight interface ("surface tension") whereas $U$ gives the energy cost for the interface having zero height. The model can be used for example to describe the formation of a new layer of atoms on a solid substrate. The probability (2) can be recast into

$$
P\left(\left\{m_{i}\right\}\right)=\prod_{i=1}^{L} g\left(m_{i}, m_{i+1}\right),
$$

where $g(m, n)=\exp \left[-J|m-n|+(U / 2)\left(\delta_{m, 0}+\delta_{n, 0}\right)\right]$ is the pairwise weight. Although this model is an equilibrium model, more recently a class of nonequilibrium models which exhibit such steady states have also been proposed [16]. One could hope that if conditions (14), (15) are not satisfied there might be some choices of rates $u(m, n)$ which yield pair-factorised states. We shall show that actually this is not the case and that either (14), (15) is satisfied or the steady state has an unknown form.

\section{Misanthrope process}

We now define the model that we consider. $M$ particles reside on sites of a onedimensional closed, periodic chain of length $L$. Each site $i$ carries $m_{i}$ particles, so that $\sum_{i=1}^{L} m_{i}=M$. A particle hops from site $i$ to site $i+1$ with rate $u\left(m_{i}, m_{i+1}\right)$ which depends on the occupancies of both the departure and the arrival site, see Fig. 1 . Periodicity implies $m_{L+1}=m_{1}$.

The misanthrope process can be mapped to the total asymmetric simple exclusion process (TASEP) on a closed chain. In Fig. 2 we show how this can be done. In TASEP, each site of a periodic 1d lattice is occupied by at most one particle. We can identify the 
number of particles in the misanthrope process with the number of vacancies between the particles in TASEP. To obtain the same dynamics as in the misanthrope process, we assume that the particle jumps to the left with rate $u(m, n)$ depending on the number of empty sites in front $(m)$ and behind $(n)$ the particle.

It is known that under certain conditions the steady state probability of the misanthrope process is given by a factorised form

$$
P\left(\left\{m_{i}\right\}\right)=\frac{1}{Z_{L}} \prod_{i=1}^{L} f\left(m_{i}\right) \delta_{M, \sum_{j} m_{j}},
$$

where $f(m)$ is a certain non-negative weight function and $Z_{L}$ is the normalization

$$
Z_{L}=\sum_{\left\{m_{i}=0\right\}}^{\infty} \prod_{i=1}^{L} f\left(m_{i}\right) \delta_{M, \sum_{j} m_{j}}
$$

In (41) the Kronecker delta imposes the constraint that the total mass (number of particles) $M$ in the system is conserved. The conditions for factorisation can be derived as follows. In the steady state the equation balancing probability currents from and to a given configuration is

$$
\begin{aligned}
& \sum_{i=1}^{L} u\left(m_{i}, m_{i+1}\right) P\left(\ldots, m_{i-1}, m_{i}, \ldots\right)= \\
& \sum_{i=1}^{L} u\left(m_{i-1}+1, m_{i}-1\right) P\left(\ldots, m_{i-1}+1, m_{i}-1, \ldots\right),
\end{aligned}
$$

where $P\left(\left\{m_{i}\right\}\right)$ is assumed to be given by Eq. (41). Here we have taken the conventions that $u(0, n)=0$ (the hop rate is zero if there are no particles at the departure site), $u(m,-1)=0$, and $m_{0}:=m_{L}, m_{L+1}:=m_{1}$. Dividing both sides by $\prod_{i=1}^{L} f\left(m_{i}\right)$ and shifting indices $(i-1, i) \rightarrow(i, i+1)$ in terms on the right-hand, we obtain

$$
\sum_{i=1}^{L}\left[u\left(m_{i}, m_{i+1}\right)-u\left(m_{i}+1, m_{i+1}-1\right) \frac{f\left(m_{i}+1\right) f\left(m_{i+1}-1\right)}{f\left(m_{i}\right) f\left(m_{i+1}\right)}\right]=0 .
$$

In Appendix A we show that the most general solution to an equation of the form

$$
\sum_{i=1}^{L} F\left(m_{i}, m_{i+1}\right)=0
$$

reads $F(a, b)=h(a)-h(b)$ for $L>2$. Therefore, from Eq. (17) we obtain that

$$
u(m, n)-u(m+1, n-1) \frac{f(m+1) f(n-1)}{f(m) f(n)}=h(m)-h(n),
$$

with some (yet unknown) function $h(m)$. Let us consider now the case $n=0$. Because $u(m+1,-1)$ is identically zero, the second term in the above equation vanishes and we obtain

$$
h(m)=u(m, 0)+h(0) .
$$


Similarly, for $m=0$ and arbitrary $n$, the first term of Eq. (9) vanishes and we find

$$
-u(1, n-1) \frac{f(1) f(n-1)}{f(0) f(n)}=h(0)-h(n)=-u(n, 0) \text {. }
$$

The last relation (11) may be rearranged to give a recursion relation for $f(n)$ as follows

$$
f(n)=f(n-1) \frac{f(1)}{f(0)} \frac{u(1, n-1)}{u(n, 0)} .
$$

Inserting now (10) and (12) into Eq. (91) we arrive at the constraint on $u(m, n)$ which allows for the factorized steady state:

$$
u(m, n)=u(m+1, n-1) \frac{u(1, m) u(n, 0)}{u(m+1,0) u(1, n-1)}+u(m, 0)-u(n, 0) .
$$

It can be proved (see Appendix B) that this actually reduces to two conditions:

$$
\begin{aligned}
& u(n, m)=u(m+1, n-1) \frac{u(1, m) u(n, 0)}{u(m+1,0) u(1, n-1)}, \\
& u(n, m)-u(m, n)=u(n, 0)-u(m, 0) .
\end{aligned}
$$

These conditions were first written down by Cocozza-Thivent [9] and can also be derived by using the condition of dynamical reversibility [17]. Alternative proofs are presented in [10] and [18].

To obtain insight into the physical meaning of conditions (14), let us examine the matrix $u_{m n} \equiv u(m, n)$. The first row is made up of zeros since $u_{0 n}=u(0, n)=0$. Let us define two vectors $\vec{x}=\left\{x_{1}, x_{2}, \ldots\right\}$ and $\vec{y}=\left\{y_{1}, y_{2}, \ldots\right\}$ of infinite length which specify the second row and the first column, i.e.,

$$
\begin{aligned}
& u(m, 0)=y_{m}, \\
& u(1, n)=x_{n} .
\end{aligned}
$$

The matrix $u_{m n}$ assumes now the following form:

$$
u=\left[\begin{array}{ccccc}
0 & 0 & 0 & 0 & \ldots \\
y_{1} & x_{1} & x_{2} & x_{3} & \ldots \\
y_{2} & . & . & . & \\
y_{3} & . & . & . & \\
\vdots & & & &
\end{array}\right] .
$$

Equations (14) and (15) can now be rewritten as two recursion relations which allow one to find $u(m, n)$ for $m>2, n>1$ :

$$
\begin{aligned}
& u(m+1, n-1)=u(n, m) \frac{y_{m+1}}{x_{m}} \frac{x_{n-1}}{y_{n}}, \\
& u(n, m)=u(m, n)-y_{m}+y_{n} .
\end{aligned}
$$

By iterating these equations one obtains unambiguous expressions for all $u(m, n)$, for example, given $u(1, n)$ for $n>0$, (20) implies $u(n, 1)$ and (19) gives $u(2, n-1)$. Thus the hop rate is fully determined by fixing the rate $y_{m}$ of hopping from a site with $m$ particles to an empty site, and the rate $x_{n}$ of hopping from a site with only one particle to a site 
with $n$ particles. There is, however, an additional condition that $u(m, n)$ must be nonnegative for all $m, n$. This imposes some constraints on $y_{m}, x_{n}$ which we were not able to express in a closed form and hence we do not have an expression for the most general form of $y_{m}, x_{n}$ that implies non-negative $u(m, n)$. In what follows we will consider some special cases of $y_{m}, x_{n}$ for which one can prove non-negativeness of $u(m, n)$.

Let us now derive a formula for the weight function $f(n)$. Iterating Eq. (12) and using the definitions of $y_{m}, x_{n}$ (16,17) we obtain

$$
f(n)=f(0)\left(\frac{f(1)}{f(0)}\right)^{n} \prod_{i=1}^{n} \frac{x_{i-1}}{y_{i}} .
$$

It is important to note that any exponential factor $A q^{n}$ in $f(n)$ does not change the steady-state properties since it appears in $P\left(m_{1}, \ldots, m_{L}\right)$ as a constant prefactor

$A^{L} q^{\sum_{i} m_{i}}=A^{L} q^{M}$ due to the fixed number of sites, $L$, and particles $M=\sum_{i} m_{i}$. Thus we may neglect the factor $f(0)(f(1) / f(0))^{n}$ in Eq. (21) and write

$$
f(n)=\prod_{i=1}^{n} \frac{x_{i-1}}{y_{i}} .
$$

\subsection{Lack of Pair-factorized states}

One may wonder whether conditions (14]15) could be relaxed if, for example, instead of insisting on the factorization of the steady state over sites we assumed a much lessrestrictive factorization over pairs of sites (Eq. (3) ) as in Ref. [16, 19]. There, the hop rate depended on the occupation number $m_{i}$ of the departure site as well as two neighbouring sites $m_{i-1}, m_{i+1}$. Surprisingly, it turns out that for the misanthrope process, whose hop rate depends only on the departure and arrival site, pair-factorization is not possible except for a trivial case when the steady state fully factorizes as in Eq. (4). We give a proof of this result in Appendix C.

\section{Physical examples of the hop rate}

In this section we present some particular solutions to (14,15) or equivalently (19,20) which represent physical models, in the sense that the hop rates are non-negative.

\subsection{Factorized hop rate}

One can check that for a factorized hop rate of the form

$$
u(m, n)=w(m) v(n),
$$

equation (14) is automatically fulfilled. When $v(n)=$ constant, equation (15) is also fulfilled and we recover the ZRP with $u(m, n)=w(m)$. When $v(n) \neq$ constant, equation (15) leads to the relation between $w(n)$ and $v(n)$ :

$$
w(n)=C[v(n)-v(0)],
$$


with some arbitrary, non-zero constant $C$. Thus

$$
u(m, n)=C[v(m)-v(0)] v(n)
$$

is the form of a factorised hop rate that yields a factorised steady state. For nonnegativeness of $u(m, n)$, it suffices that one of the following conditions is fulfilled:

(i) $C>0, v(m)>v(0)>0$ for $m>0$,

(ii) $C<0, v(0)>v(m)>0$.

In both these cases $v(m)$ is always positive. One could additionally consider cases where $v(m)$ is always negative but as $u(m, n)$ is invariant under $v \rightarrow-v$ these are trivially equivalent to the two cases above.

Our first simple example of the factorised hop rate (25) is $C=-1$ and $v(n)=N-n$ with some integer $N>0$. This leads to

$$
u(m, n)=m(N-n) .
$$

If $N=1$ this reduces to the asymmetric simple exclusion process where the occupancy of each site is limited to 1 and $u(1,1)=0$. Similarly the case of general integer $N>0$ corresponds to 'partial exclusion' [20] where each site of a lattice contains at most $N$ particles and each particle attempts hops forward to the next site with rate one which succeed with probability $N-n$ where $n$ is the occupancy of the destination site.

The case $v(m)=m$ for $m>0$ has been studied and is referred to as the Inclusion Process. In a prescribed limit $v(0) \rightarrow 0$, the limit of zero hopping rate onto empty sites, a form of condensation occurs [21].

\subsection{Non-factorized choices of $u(m, n)$}

The factorized form (25) is not the only allowed form for $u(m, n)$ that solves (19,20) and gives rise to a factorized steady state. To see this, let us consider an example in which $x_{n}=y_{n+1}$, with $y_{n}>0$. In this case, one can check that

$$
u(m, n)=\sum_{i=0}^{n} y_{m+i}-\sum_{i=1}^{n} y_{i}
$$

is the solution to equations (19), (20) . To ensure that the hop rate is positive, a sufficient condition is that $y_{n}$ grows with $n$.

Another interesting example is when $y_{m}=y=$ const $>0$ and $x_{n}$ is positive but otherwise arbitrary. Then (20) implies that $u(n, m)=u(m, n)$ and (19) can be iterated to yield for $m>0, n>1$

$$
u(m, n)=\frac{\prod_{i=m}^{m+n-1} x_{i}}{\prod_{j=1}^{n-1} x_{j}} .
$$

The corresponding weight function (22) reads for $n>1$

$$
f(n)=y^{-n} \prod_{i=1}^{n-1} x_{i} \rightarrow \prod_{i=1}^{n-1} x_{i}
$$


where in the last expression we have suppressed a term exponential in $n$ which as, previously noted, is unimportant. This expression assumes a form similar to the weight function in the zero-range process. In fact, this model is closely related to a recently considered facilitated exclusion process [22]. In that work an exclusion process is considered wherein, for a particle to hop, the neighbouring site behind has to be occupied. Using the standard mapping of section 1 (see Fig. 2) the facilitated exclusion process corresponds to the limit $u(m, 0)=y=1$ and $u(m, n)=x \rightarrow 0$ of the misanthrope process.

\section{Condensation in the misanthrope process}

An important feature of models such as ZRP [10] or balls-in-boxes model [23], in which the steady-state probability takes the factorized form (4), is that for some choices of the weight function $f(n)$ the system exhibits a phase transition in the thermodynamic limit $M, L \rightarrow \infty$. If the density of particles $\rho=M / L$ is above some critical value $\rho_{c}$, the surplus of particles $M-\rho_{c} L$ accumulates at a single site and is called the condensate. The sufficient condition for the condensation above some finite $\rho_{c}$ is the appropriate asymptotic behaviour of $f(n)$ [10]. As any exponential dependence in $f(n)$ is irrelevant (see previous section), we just need to consider the asymptotic behaviour of $f(n)$ modulo any exponential factor. There are two generic cases (see e.g. [24]):

I $f(m) \sim m^{-\gamma}$ with $\gamma>2$. The critical density is finite but its numerical value depends on the particular form of $f(m)$ and not only on its asymptotic behaviour. The fraction $\rho / \rho_{c}-1$ of all particles goes to the condensate. We will refer to this as standard condensation.

II $f(m)$ increases with $m$ more quickly than exponentially, e.g., as $\sim m$ !. This leads to so called strong (or complete) condensation - the critical density $\rho_{c}=0$ and a fraction of particles tending to one in the thermodynamic limit is located at one site.

There are also some other specific examples, such as the Backgammon model [25, 26], which corresponds to taking $f(m)=e^{\beta \delta_{m_{i}, 0}}$ and exhibits condensation in the limit $\beta \rightarrow \infty$, and the inclusion process in the limit $v(0) \rightarrow 0$ mentioned earlier [21].

To see why the condensation happens in the two generic cases highlighted above, we shall follow a standard approach [10]. Treating the steady-state probability as the statistical weight of a given configuration, and defining the grand-canonical partition function

$$
G(z)=\sum_{\left\{m_{i}\right\}} \prod_{i=1}^{L} f\left(m_{i}\right) z^{m_{i}}=F(z)^{L}
$$

where

$$
F(z)=\sum_{m=0}^{\infty} f(m) z^{m}
$$


we see that the phase transition, signalled by a singularity of $G(z)$ at some $z_{c}$, is possible only if the series $F(z)$ has a finite radius of convergence $z_{c}$. Moreover, the density calculated as a function of fugacity $z$ from the grand-canonical partition function,

$$
\rho=z \frac{F^{\prime}(z)}{F(z)}
$$

must be finite as $z \rightarrow z_{c}^{-}$. This is only possible if $f(m)$ decays as a power law in which case we may have a finite $\rho_{c}$ (case I) or $f(m)$ grows very fast with $m$ in which case $z_{c}=0$ and $\rho_{c}=0$ (case II). Any exponential factor in $f(m)$ only shifts the position of $z_{c}$.

\subsection{Factorized hop rate}

We will now discuss which choices of the hop rate $u(m, n)$ of the misanthrope process lead to the generic cases I, II described above. We begin by considering factorized hop rates $u(m, n)$, as in Eq. (25). Using

$$
x_{n}=v(n)(v(1)-v(0)), \quad y_{m}=v(0)(v(m)-v(0)),
$$

equation (22) yields

$$
\frac{f(m)}{f(m-1)} \propto \frac{v(m-1)}{v(m)-v(0)},
$$

where we have removed a constant factor that generates an exponential factor in $f(m)$. Let us first consider strong condensation (case II), that is $f(m) / f(m-1) \rightarrow \infty$ for large $m$. From Eq. (34) we see that for this to happen, $v(m)$ has to tend to $v(0)$ in the limit of $m \rightarrow \infty$. For example, for

$$
v(0)=1, \quad v(m)=1+\frac{1}{m+1},
$$

we obtain $f(m) / f(m-1)=(m+1)^{2} / m$ and strong condensation occurs.

In standard condensation (case $\mathbf{I}$ ) we require a power law decaying as

$$
f(m) \sim m^{-\gamma},
$$

so that

$$
\frac{f(m)}{f(m-1)} \sim 1-\frac{\gamma}{m}
$$

Expression (34) fulfils this condition for two distinct large $m$, asymptotic behaviours of $v(m)$ :

$$
v(m) \sim m^{\gamma}
$$

and

$$
v(m) \cong \beta\left(1-\frac{\alpha}{m}\right)
$$

Clearly, the different hop rates (38,39) constructed from the different choices for $v(m)$ will lead to very different dynamical properties system which we shall study in detail in Section 5 , 
For the first case (38), condensation is possible for any $\gamma>2$. The exact form of $v(m)$ is not important. For the second case (39), we obtain from Eq. (34) that

$$
\frac{f(m)}{f(m-1)} \simeq A\left(1-\frac{\alpha v(0)}{m(v(0)-\beta)}\right)
$$

where $A$ is a constant. Therefore the asymptotic behaviour of the single-site weight is $f(m) \simeq A^{m} m^{-\gamma}$ where the exponent $\gamma$ reads

$$
\gamma=\alpha \frac{v(0)}{v(0)-\beta} .
$$

Condensation is possible if $\gamma$ is larger than 2. This leads to the following conditions on $\beta, v(0)$ and $\alpha$ for condensation to occur:

$$
\begin{aligned}
& \text { for } \beta<v(0): \alpha>\frac{2(v(0)-\beta)}{v(0)}, \\
& \text { for } \beta>v(0): \alpha<-\frac{2(\beta-v(0))}{v(0)} .
\end{aligned}
$$

Interestingly, the existence of condensation depends not only on the asymptotic behaviour of $v(m)$ but also on $v(0)$. This should be contrasted with the ZRP for which it is only the asymptotic decay of the hop rate, given by $\alpha$ in $u(m) \cong \beta(1+\alpha / m)$, that determines whether condensation is possible or not and the exact form of $u(m)$ affects only the value of the critical density, above which condensation occurs. Here, the influence of the particular form of $v(m)$ is much more significant.

\subsection{Illustrative example of a factorized hop rate}

As an illustrative example consider the hop rate

$$
v(0)<1, \quad v(m)=1+\frac{1}{m+1},
$$

which differs from Eq. (35) only in the value of $v(0)$. In the previous case, $v(0)=1$ we have strong condensation, whereas $v(0)<1$ in Eq. (44) corresponds to $\alpha=-1, \beta=1$ from Eq. (39). Thus, by (43), for $v(0) \leq 2 / 3$ there is no condensation, but for $2 / 3<v(0)<1$ there is standard condensation and for $v(0)=1$ there is strong condensation, even though $v(m)$ is the same in all cases for $m>0$.

It is possible to compute exactly the critical density in this case by using (22), which yields

$$
f(n)=\frac{(n+1) !(n+1) !}{n !(c)_{n}},
$$

after removing exponential factors in $n$. Here

$$
c=\frac{3-2 v(0)}{1-v(0)},
$$

and we use the Pochhammer symbol $(c)_{n}=c(c+1) \ldots(c+n-1)$. Then the generating function (31) is a hypergeometric function

$$
F(z)=\sum_{n=0}^{\infty} \frac{(n+1) !(n+1) !}{n !(c)_{n}} z^{n}={ }_{2} F_{1}(2,2, c ; z),
$$


which converges for $z \leq 1$. Using standard identities for the hypergeometric function (which hold when the series converge) [27],

$$
\begin{aligned}
& { }_{2} F_{1}(a, b, c ; 1)=\frac{\Gamma(c) \Gamma(c-a-b)}{\Gamma(c-a) \Gamma(c-b)}, \\
& \frac{\partial}{\partial z}{ }_{2} F_{1}(a, b, c ; z)=\frac{a b}{c}{ }_{2} F_{1}(a+1, b+1, c+1 ; z),
\end{aligned}
$$

one may determine the critical density as the maximum allowed density (32), given by $z \rightarrow 1:$

$$
\rho_{c}=\left.\frac{z F^{\prime}(z)}{F(z)}\right|_{z=1}=\frac{4}{c} \frac{{ }_{2} F_{1}(3,3, c+1 ; 1)}{{ }_{2} F_{1}(2,2, c ; 1)}=\frac{4}{c-5}=\frac{4(1-v(0))}{3 v(0)-2} .
$$

We see indeed that $\rho_{c} \rightarrow 0$ as $v(0) \rightarrow 1$ and $\rho_{c} \rightarrow \infty$ as $v(0) \rightarrow 2 / 3$.

\subsection{General case with arbitrary $x_{m}, y_{n}$}

We now turn to the case of general $u(m, n)$ satisfying (14),15). From Eq. (21) we obtain that

$$
\frac{f(m)}{f(m-1)}=\frac{x_{m-1}}{y_{m}}
$$

In order to have strong condensation (case II) we require $x_{m-1} / y_{m} \rightarrow \infty$ for large $m$. For standard condensation with $f(m) \sim m^{-\gamma}$ (36) (case I) we have again two distinct possible asymptotic behaviours for $x_{m}, y_{n}$ :

$$
y_{n} \cong 1+\frac{A}{n}, \quad x_{m} \cong 1+\frac{B}{m},
$$

which gives $\gamma=A-B$, and $A-B>2$ as the necessary condition for condensation, and

$$
y_{n} \sim(n+C)^{\alpha}, \quad x_{m} \sim(m+D)^{\alpha},
$$

which in turn leads to $\gamma=\alpha(1+C-D)$. The condition for condensation reads now $\alpha(1+C-D)>2$. As before, the two asymptotic forms of hop rates (52) and (53) lead to different dynamics.

\section{Dynamics of the condensate}

We return now to the different microscopic dynamics that yield the same steady state and exhibit condensation above the same $\rho_{c}$. To gain insight into the dynamics, we simulated this system using a modified continuous time Monte Carlo in which the time interval to the next event is stochastically generated. We prepare the system in a state in which particles are randomly distributed among the sites. Then, in each time step we choose a random site $i$ and move a particle from this site to its right neighbour. The site is selected at random with probability $u\left(m_{i}, m_{i+1}\right) / \sum_{j} u\left(m_{j}, m_{j+1}\right)$. To do this effectively, we sort all possible "moves" with respect to their probabilities of occurrence. Lastly, we update the time $t \rightarrow t+\Delta t$ by adding an exponentially distributed random variable $\Delta t$ with mean $1 / \sum_{j} u\left(m_{j}, m_{j+1}\right)$. To minimize computational time, after each 
move we recalculate only the rates $u\left(m_{i-1}, m_{i}\right), u\left(m_{i}, m_{i+1}\right)$, and $u\left(m_{i+1}, m_{i+2}\right)$ which have been affected by the move. We also use tabulated values of $u(m, n)$ to avoid time-consuming algebraic operations such as calculating $m^{\gamma}$ for non-integer $\gamma$.

\subsection{ZRP-like rates given by Equation (39)}

In Figure 3 we show a graph for a typical simulation run for ZRP-like hop rates (39), that is

$$
v(m)=\beta\left(1-\frac{\alpha}{m}\right) \quad \text { for } \quad m>0,
$$

and $v(0)>\beta$. Then taking $C=-1$ in (25), yields the asymptotic behaviour

$$
u(m, n) \cong \beta(v(0)-\beta)-\frac{\alpha \beta(v(0)-\beta)}{n}+\frac{\alpha \beta^{2}}{m},
$$

for $m, n$ sufficiently large. In this figure, the positions of the five most-occupied sites are plotted against time, together with snapshots of the system at three different times. We see that at first, small condensates are formed (multiple horizontal lines in the upper diagram). These condensates coalesce quickly until only two are left. Finally, the two condensates merge into a single one; this last process is the slowest. The size of the condensate initially grows quickly and then slows down, see Fig. 4, left. This is very similar to the condensation in the zero-range process with $u(m)=1+\gamma / m$, which also leads to the power-law $f(m) \sim m^{-\gamma}$. The dynamics of such ZRP has been extensively studied in the past [28, 29]. It has been argued that the typical time to reach the steady state in an asymmetric 1d system scales as $L^{2}$ with the number of sites $L$ if the density $\rho=M / L$ is kept constant and $M, L$ are sufficiently large.

One can argue that the same scaling should hold in our system, that is the $n$ dependence of $u(m, n)$ in Eq. (55) is not important. The argument goes as follows. In the last, slowest stage of condensation when only two condensates are left, occupation numbers on sites surrounding these condensates fluctuate very rapidly. Thus, from each condensate's point of view, the hop rates $u\left(m_{i-1}, m_{i}\right), u\left(m_{i}, m_{i+1}\right)$ to and from the condensate at site $i$ are effectively averaged over $m_{i-1}, m_{i+1}$. According to formula (55), the average inflow rate is $\left\langle u\left(m_{i-1}, m_{i}\right)\right\rangle \cong$ const $-\frac{\alpha \beta(v(0)-\beta)}{m_{i}}$ while the outflow rate reads $\left\langle u\left(m_{i}, m_{i+1}\right)\right\rangle \cong$ const $+\frac{\alpha \beta^{2}}{m_{i}}$. The net flow between both condensates having $m_{1}$ and $m_{2}$ particles, respectively, is $\sim\left(1 / m_{1}-1 / m_{2}\right)$. Since both condensates have $O(L)$ particles, the flow is at most $O\left(L^{-1}\right)$. Thus, statistical fluctuations which are of order $u\left(m_{i}, m_{i+1}\right) \sim u\left(m_{i-1}, m_{i}\right) \sim O(1)$ dominate and $m_{1}, m_{2}$ perform effectively unbiased random walks, at least as long as they are of order $O(L)$. The typical time scale is defined by the time it takes to reach either $m_{1}=0$ or $m_{2}=0$, which scales diffusively as $T \sim L^{2}$. The finite distance $O(L)$ between the condensates does not matter, because due to the finite average hop rate in the bulk, the particles traverse it in $\sim L$ steps which is smaller than the time scale related to fluctuations.

To confirm this prediction, we carried out simulations for $\rho \gg \rho_{c}$ and various sizes $L$, and measured the time $T$ it took before the maximal occupation number reached 

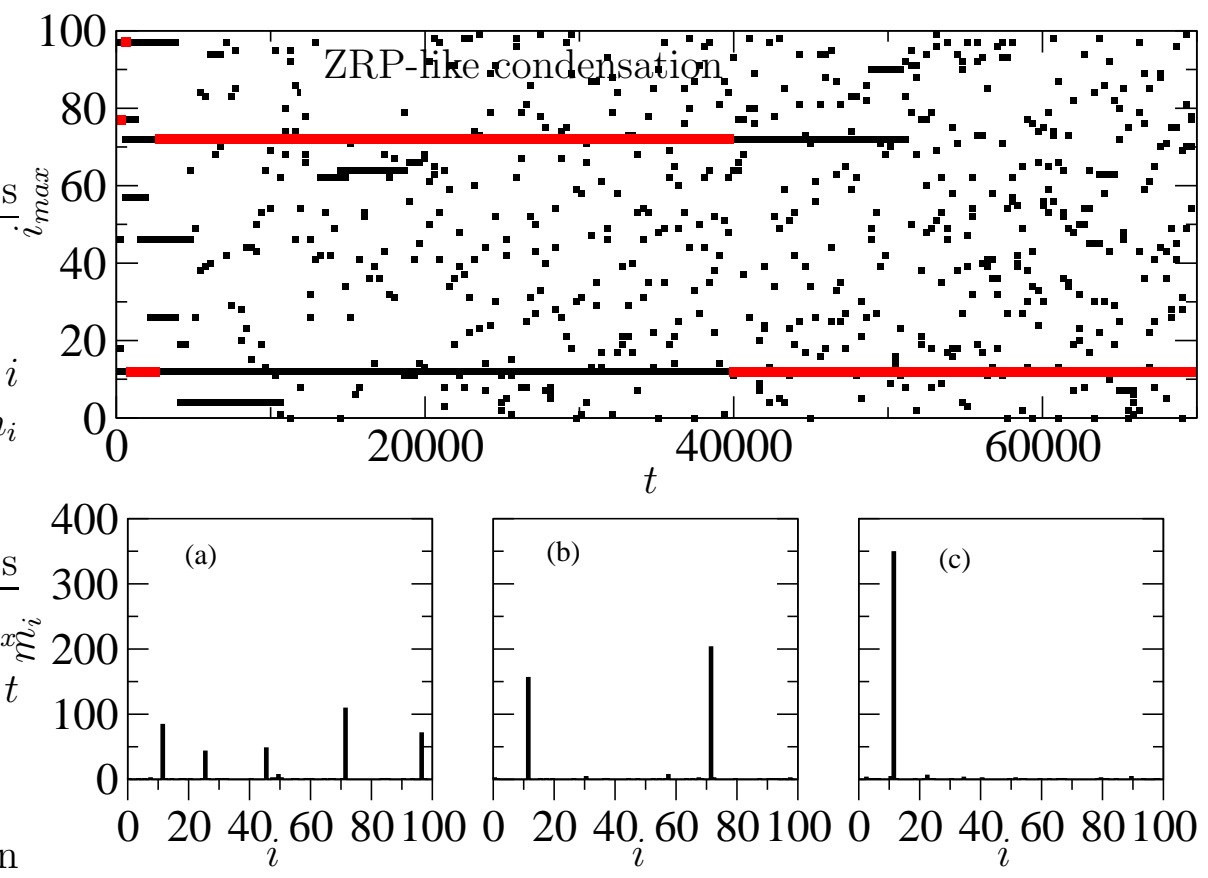

Figure 3. Top: positions of five most occupied sites as a function of time (squares) in a system with $L=100$ sites, $M=400$ particles, and the factorized hop rate with $v(m)=\beta\left(1-\frac{\alpha}{m}\right)$ defined in Eq. (39) for $v(0)=1.3, \alpha=0.7, \beta=1$ (this corresponds to $\gamma \approx 3.03)$. Red squares show the position of the site with the maximal number of particles (the condensate). Bottom: three snapshots of the system for a) $t=2800$, b) $t=30000$, c) $t=80000$. After a fast nucleation stage during which many small condensates are formed, the condensates slowly merge into a single one.
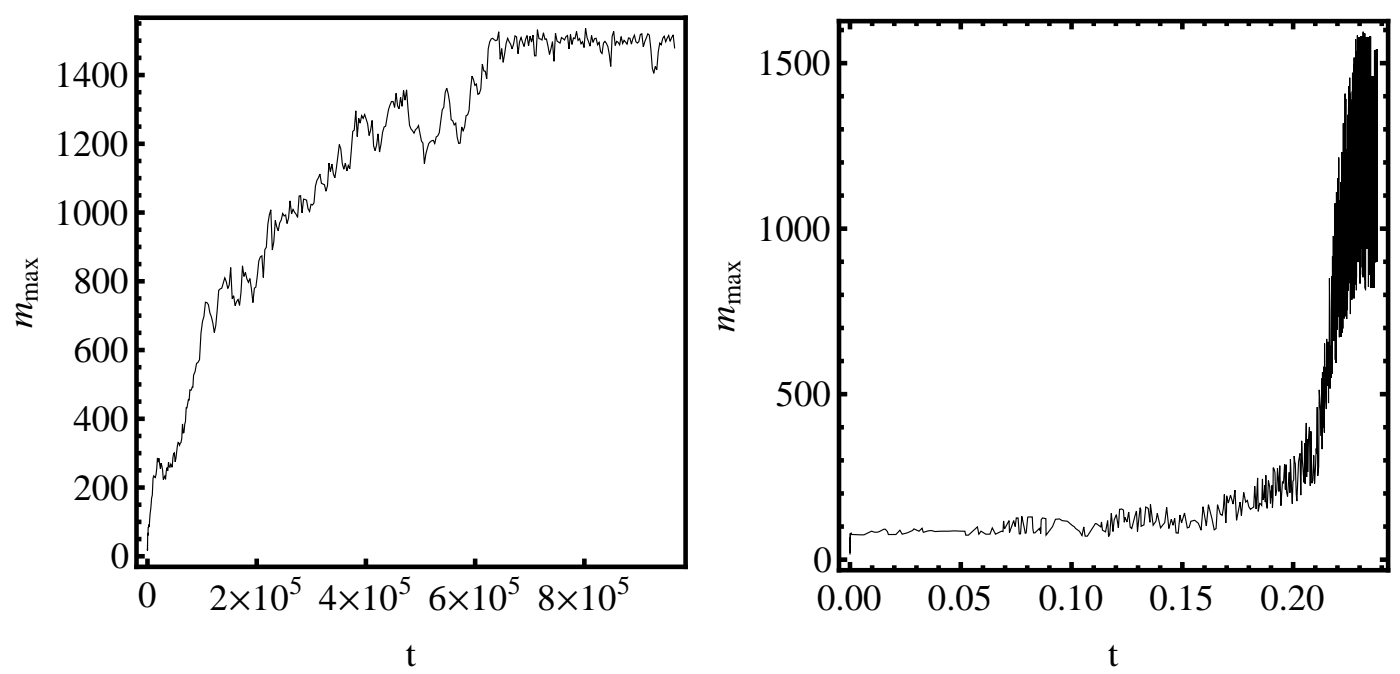

Figure 4. Simulation runs show the occupation $m_{\max }$ of the site with maximal number of particles as a function of time $t$. Left: ZRP-like condensation $(v(0)=1.3, \alpha=$ $0.7, \beta=1$ in Eq. (39)), right: explosive condensation with $v(m)=(m+0.1)^{3} . L=200$, $M=1600$ (density $\rho=8$ ) in both cases. 

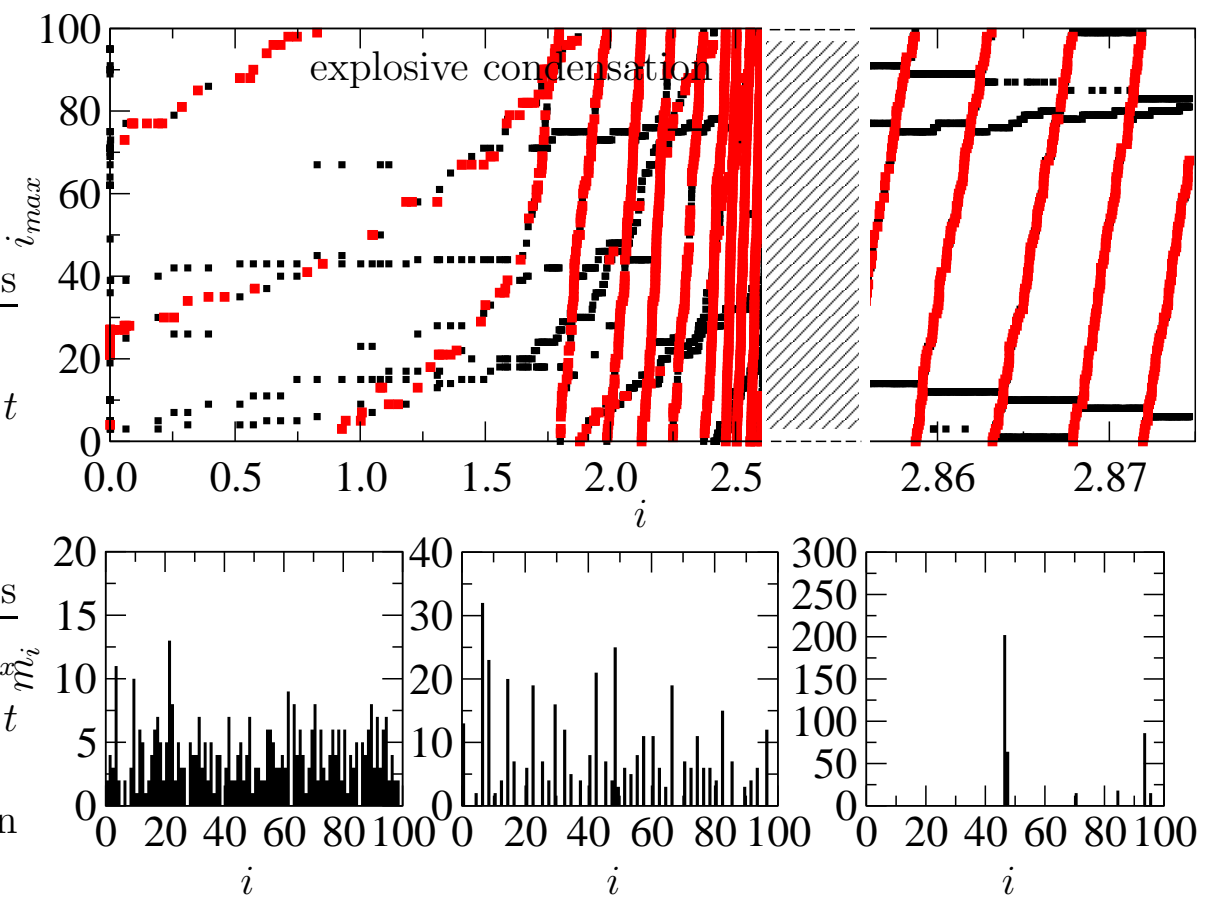

Figure 5. Top: positions of five most occupied sites (as in Fig. 3) as a function of time (squares) in a system with $L=100$ sites, $M=400$ particles, and the factorized hop rate $v(m)=(m+0.1)^{3}$ as in Eq. (38) for $\gamma=3$. Red squares show the position of the condensate. Bottom: three snapshots of the system for a) $t=0, \mathrm{~b}) t \approx 1, \mathrm{c}$ ) $t \approx 2.86$. Initial nucleation is the slowest process, followed by a rapid growth of the condensate which "sucks up" particles as it moves at increasing velocity to the right. The velocity of the condensate can be read off from the slope of the red line in the top figure. The terminal velocity of the condensate in the steady state (not shown here) is $\approx(0.1 M)^{3} \approx 64000$.

the mean steady-state value $M-L \rho_{c}$. One clearly sees from Fig. 6, left, that $T$ scales approximately as $\sim L^{2}$, independently of $\gamma$ and other parameters.

\subsection{Explosive-condensation rates given by Equation (38)}

We now turn to the case $v(m) \sim m^{\gamma}$ from Eq. (38), for which $u(m, n) \sim(m n)^{\gamma}$ for large $m, n$. To be more specific, we choose $v(m)=(\epsilon+m)^{\gamma}$ so that

$$
u(m, n)=\left((\epsilon+m)^{\gamma}-\epsilon^{\gamma}\right)(n+\epsilon)^{\gamma},
$$

with (typically) $\epsilon \ll 1$. In Fig. 5 we show results of computer simulations. If we compare it with Fig. 3, we see some striking differences. First, the site carrying the maximal number of particles moves unidirectionally, in the same direction as hopping particles. The motion of the condensate is similar to the "slinky"-like motion of a nonMarkovian model [30, 31] or a generalization of ZRP to non-factorizing probabilities 32. Second, the evolution speeds up in time; the condensate moves faster and faster as it gains particles, see also Fig. 4, right. This is why in the previous work [12] we termed it "explosive condensation". Third, the speed at which the condensate travels through 



Figure 6. Left: time to reach the steady state as a function of the system size $L$, for $\rho=4, \beta=1, \alpha=0.7$ and for $v(0)=1.3$ (circles) and $v(0)=1.21$ (squares), which correspond to $\gamma \approx 3, \rho_{c} \approx 0.5$ and $\gamma \approx 4, \rho_{c} \approx 0.3$, respectively. Thick line corresponds to a theoretical prediction $T \sim L^{2}$. Right, the same plot for $v(m)$ from Eq. (56) (explosive condensation) with $\epsilon=1$ and particle density $\rho=8$. Curves from top to bottom: $\gamma=3,4,5$.

the system stabilizes after the system reaches the steady state. Finally, other (smaller) peaks move in the opposite direction to the main condensate each time they interact with it.

The dynamics thus differs significantly from the ZRP-like case. Figure 6, right, shows another striking feature of this new condensation. If we fix the density of particles $\rho$ and increase the system size $L$, the time $T_{\mathrm{ss}}$ to reach steady state decreases as a function of $L$. This is in contrast to the ZRP-like behaviour from Fig. 6, left, and to the ordinary ZRP, where the time to steady state increases with $L$. In Ref. [12] we focused on deriving the relation between $T_{\mathrm{ss}}$ and $L$, assuming that the time scale $T_{\mathrm{ss}}$ was dominated by the slow process of initial coalescence, in contrast to the ZRP where this process is the fastest one. Our reasoning was based on several assumptions on how clusters of particles interact, which we inferred from numerical simulations. In what follows we shall provide more evidence in support of these arguments.

First, we show that a cluster of particles of size $m$ moves through the system at speed $\approx(\epsilon m)^{\gamma}$. As it moves, the cluster collides with other clusters and exchanges particles with them. On average, particles flow from smaller to larger clusters. Next, we show that the rate of accumulation increases with increasing speed of a cluster and, as a result, it takes a finite time for the cluster to reach macroscopically large occupation (condensate). We also show that the distribution of this time is quite broad and arbitrarily short times are possible (although not very likely). Finally, we show that, since there are initially $O(L)$ clusters and each of them can potentially become the condensate in a finite time, the time to reach steady state (condensation) decreases with $L$ as a result of a simple extreme value statistics argument.

We shall now discuss our arguments in detail, assuming that $\epsilon \ll 1$. Let us first calculate the speed of an isolated cluster of $m$ particles travelling in an otherwise empty system. We assume that the cluster is initially at site $i$, so that $m_{i}=m$, and $m_{i+1}=0$. 
The only allowed transition for the cluster is to lose one particle to site $i+1$, which happens with rate $u(m, 0)$. The average time for this to happen is $t_{m}=1 / u(m, 0)$. In the second step, the condensate may lose another particle with rate $u(m-1,1)$, which will take time $t_{m-1}=1 / u(m-1,1)$. Alternatively, the particle at site $i+1$ may jump to site $i+2$, but this is much less likely because $u\left(m_{i+1}, 0\right) \propto \epsilon^{\gamma} \ll 1$ and this is much smaller than $u\left(m_{i}, m_{i+1}\right)$. The process in which site $i$ loses particles in favour of site $i+1$ will thus continue with rate $u\left(m_{i}, m-m_{i}\right)$ (with other processes having negligible rates) until $m_{i}=0$ and $m_{i+1}=m$. The average times for each step will be $t_{m}, t_{m-1}, \ldots, t_{1}$, where $t_{n}=1 / u(n, m-n)$. Therefore, the total time $\tau$ of the process will be the sum of times $t_{m}, t_{m-1}, \ldots, t_{1}$ :

$$
\langle\tau\rangle=\sum_{n=1}^{m} t_{n}=\sum_{n=1}^{m} \frac{1}{u(n, m-n)}=\sum_{n=1}^{m} \frac{1}{(m-n+\epsilon)^{\gamma}\left[(\epsilon+n)^{\gamma}-\epsilon^{\gamma}\right]} .
$$

For $\gamma>2$ which is the case of condensation and for $\epsilon \ll 1$, we can approximate the above sum by the last term only, and we obtain that $\langle\tau\rangle \approx(\epsilon m)^{-\gamma}$. This shows that a cluster with $m$ particles moves $1 /\langle\tau\rangle \approx(\epsilon m)^{\gamma}$ sites per unit time. In particular, the speed of the condensate of size $M \approx\left(\rho-\rho_{c}\right) L$ is $\left(\epsilon\left(\rho-\rho_{c}\right)\right)^{\gamma} L^{\gamma}$ and increases as a power of the system size $L$. Although in our analysis we have neglected a small probability of losing particles by the cluster as it moves through the system, this is justified for both small clusters and the macroscopic cluster (the condensate). For small clusters of size $1 \ll m \ll M$ (initial stage of condensation), the distance between the clusters is of order $O(\rho L / m)$ and the probability of losing a particle between collisions $O\left(\epsilon^{\gamma} L / m\right)$ is negligible. For the condensate $(m \approx M)$, it is possible to lose particles but as it moves through the system, the condensate will also gain particles from the background and its size will fluctuate only a little. The above formula is in excellent agreement with simulations; for example, for parameters from Fig. 5, the measured speed is $63850 \pm 100$ sites per unit time, whereas the formula $(\epsilon M)^{\gamma}$ gives 64000 .

We shall now analyse what happens in a collision between two clusters of size $m_{0}$ and $n_{0}$. The process can be conveniently studied in numerical simulations by performing "scattering experiments" in which a larger condensate $m_{0}$ collides with a slower, smaller condensate $n_{0}$, see Fig. 7. We prepare the system in a state such that $m_{0}$ and $n_{0}$ are initially located at sites $i=0$ and $i=L / 2$, respectively, and we simulate the system using the same kinetic Monte-Carlo algorithm as before until one of the condensates reaches site $i=L-1$. We then measure its mass $m_{0}^{\prime}$ and calculate the mass $\Delta m$ transferred in any collision $\downarrow$ from the smaller to the larger condensate: $\Delta m=m_{0}^{\prime}-m_{0}$. In Fig. 8 we show that although the distribution of $\Delta m$ looks almost symmetrical, it is biased toward positive values. The average $\langle\Delta m\rangle \approx 0.4$ and depends only weakly on the size of the clusters involved in the collision and on the value of $\gamma$. The average mass transfer does not also depend on the value of $\epsilon$, although $\epsilon>0$ is necessary to have a non-zero hop rate for a single particle. Thus, in our further analysis below, we shall

$\ddagger$ In principle, no collision may occur but the probability of this is vanishingly small for large enough L 

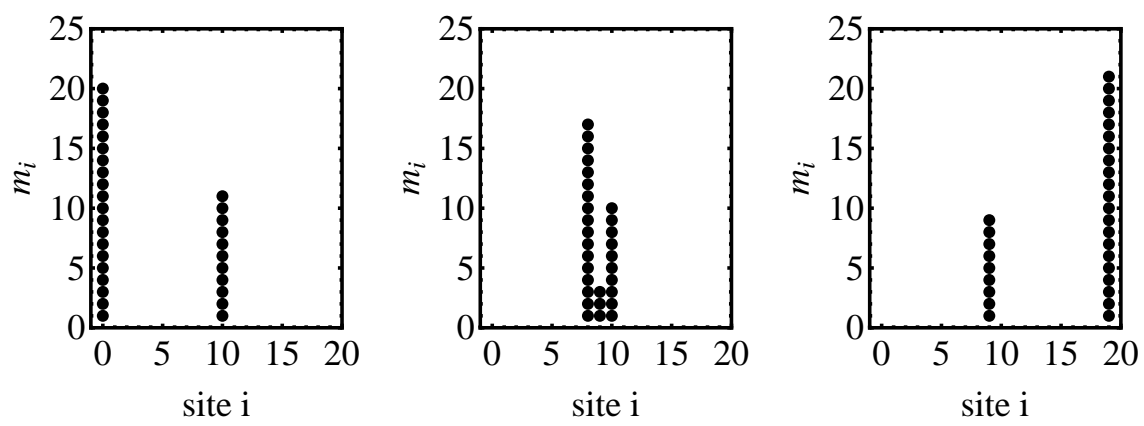

Figure 7. Example of an "experimental" setup for studying collisions of condensates. A cluster of size $m_{0}=20$ collides with a smaller cluster of size $n_{0}=10$ placed at $i=L / 2=10$. Pictures from left to right: initial state, collision, and final state at which the mass $\Delta m$ is measured at site $i=L-1=19$.
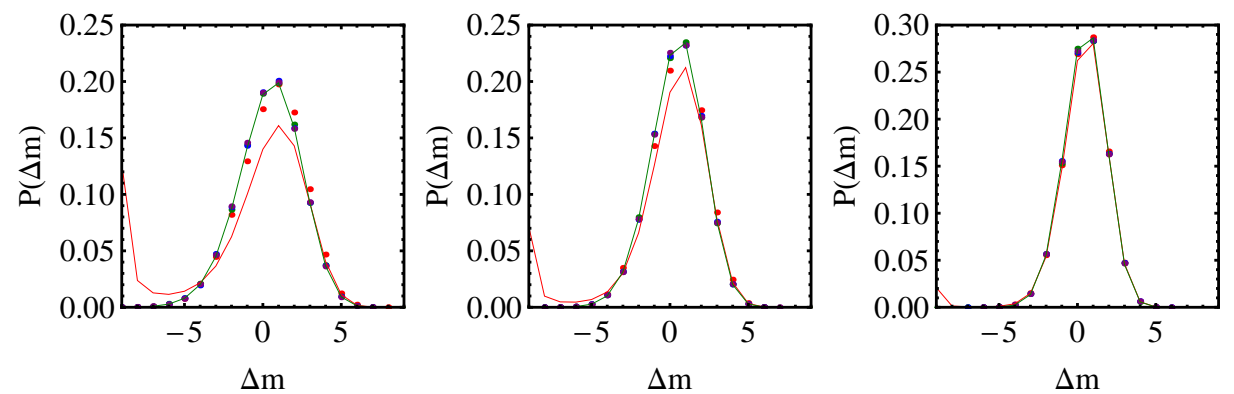

Figure 8. Plots of $P(\Delta m)$ for $\gamma=3$ (left), $\gamma=4$ (middle), and $\gamma=6$ (right). Points correspond to MC simulations for $n_{0}=10$ and $m_{0}=20,50,100,200$ (red, green, blue, and purple, respectively), whereas continuous lines are calculated using recursion (66) for $n_{0}=10$ and $m_{0}=20,50$ (red and green, respectively).

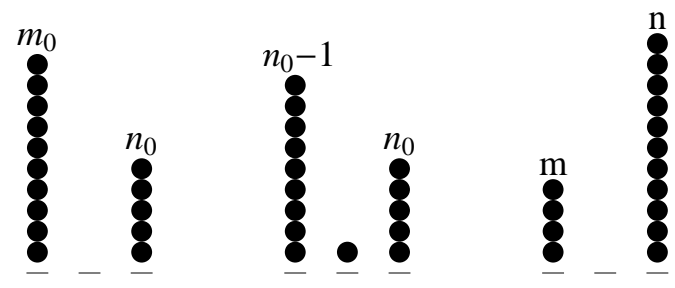

Figure 9. Collision between two clusters of sizes $m_{0}$ and $n_{0}$ (left picture) can be approximately reduced to the dynamics of only three sites. The collision is initiated when a particle hops from the left cluster to the middle site (middle picture). The collision is finished when the middle site reaches zero occupation (right picture).

assume $\epsilon \rightarrow 0$ whenever possible as this greatly simplifies calculations.

5.2.1. Deterministic description of scattering To understand the numerical results, we assume (as before) that the system is completely empty, save for the two clusters. As the larger cluster $m_{0}$ approaches the smaller cluster $n_{0}$, the collision is initiated when $m_{0}$ and $n_{0}$ become separated by just a single site with one particle that has hopped 
out from $m_{0}$, see Fig. 9. The initial configuration is therefore: $m=m_{0}-1$ particles at the left site, 1 particle at the middle site, and $n=n_{0}$ particles at the right site. In the collision process, the left site loses particles to the middle site, which in turn loses particles in favour of the right site, with the transition rates given by $u(m, M-m-n)$ and $u(M-m-n, n)$, respectively, where $M=m_{0}+n_{0}$ is the total number of particles in the system (this is a conserved quantity). As a first step, it is useful to describe the dynamics of this process using a deterministic approach in which stochasticity is neglected. Our expectation would be that this description should be valid for large clusters, for which random fluctuations of $m, n$ do not matter. The equations for the average $m, n$ read

$$
\begin{aligned}
& \dot{m}=-u(m, M-m-n)=-[m(M-m-n)]^{\gamma}, \\
& \dot{n}=u(M-m-n, n)=[n(M-m-n)]^{\gamma},
\end{aligned}
$$

with the initial condition $m(0)=m_{0}-1, n(0)=n_{0}$. We note that

$$
\frac{\dot{m}}{m^{\gamma}}+\frac{\dot{n}}{n^{\gamma}}=0
$$

which means that the quantity

$$
m^{1-\gamma}+n^{1-\gamma}=\mathrm{const}
$$

is a conserved quantity determined by the initial condition. We also note that upon time reversal $t \rightarrow$ const $-t$, the variables $m$ and $n$ exchange their roles:

$$
\begin{aligned}
& \dot{n}=-[n(M-m-n)]^{\gamma}, \\
& \dot{m}=[m(M-m-n)]^{\gamma},
\end{aligned}
$$

and thus if we change $m \rightarrow n$ and $n \rightarrow m$, we will recover Eqs. (58)-(59)). This means that the solution $(m(t), n(t))$ for $t \rightarrow \infty$ must be equal to the initial condition at $t=0$, and hence $m(t \rightarrow \infty)=n_{0}, n(t \rightarrow \infty)=m_{0}-\epsilon_{2}$ (where $\epsilon_{2}$ is small). In Fig. 10 we plot numerical solutions of Eqs. (58)-(59) which confirm our prediction. The deterministic, continuous approximations predicts zero mass transfer because the size of the larger cluster is the same as before the collision. This is at variance with the results of stochastic simulations, even for very large $m, n$, hence we see that stochasticity is important.

5.2.2. Stochastic description of scattering It is convenient to express the evolution of the fully stochastic problem not in real time $t$, but as a function the number of particles $k$ that have hopped since the beginning of the process. Assume that $k=1$ corresponds to the state $\left(m_{0}-1,1, n_{0}\right)$. From the deterministic problem and Fig. 10 we know that the occupation $M-m-n$ of the middle site first rises and then decreases again. The collision ends when the middle site reaches zero occupation, so that the final state is $\left(m, 0, m_{0}+n_{0}-m\right)$ with $m$ a random variable. The mass transferred from the smaller to the larger condensate in the collision is given by $\Delta m=\left(m_{0}+n_{0}-m\right)-m_{0}=n_{0}-m$. Let us denote by $P(m, n ; k)$ the probability 


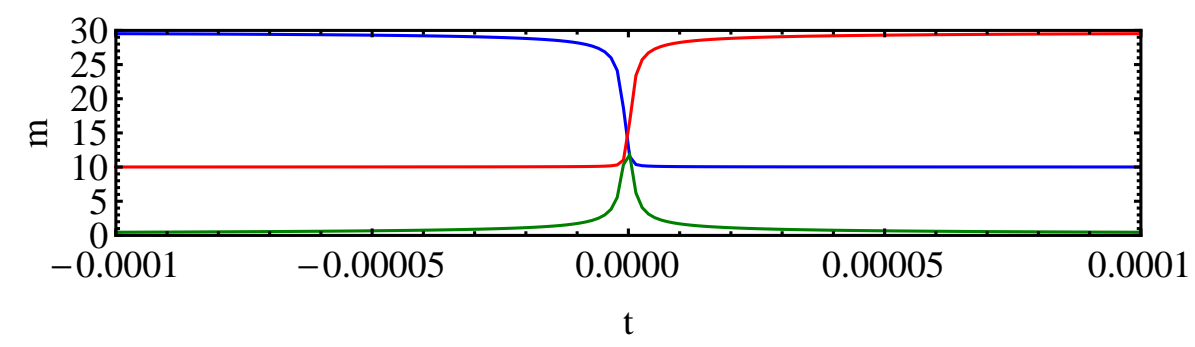

Figure 10. Solution of Eqs. (58)-(59) for $m_{0}=30-\epsilon_{2}, n_{0}=10$ and $M=m_{0}+n_{0}=40$. Curves: $m(t)$ (blue), $n(t)$ (red), and the middle-site occupation $M-m(t)-n(t)$ (green). Time axis has been shifted so that $t=0$ corresponds to the peak of the middle-site occupation $M-m-n$.

that the state of the three sites is $(m, M-m-n, n)$ after $k$ hops. To calculate the mass transferred in the process, we need to calculate

$$
\begin{aligned}
& \langle\Delta m\rangle=\sum_{m=0}^{m_{0}-1}\left(n_{0}-m\right) P\left(m, m_{0}+n_{0}-m ; 2\left(m_{0}-m\right)-1\right) \\
& =\sum_{\Delta m=n_{0}-m_{0}+1}^{n_{0}} \Delta m P(\Delta m),
\end{aligned}
$$

where $P(\Delta m)=P\left(n_{0}-\Delta m, m_{0}+\Delta m ; 2\left(m_{0}-n_{0}+\Delta m\right)-1\right)$ is the distribution of the mass transferred. The probability $P(m, n ; k)$ can be found recursively:

$$
\begin{aligned}
& P(m, n ; k)=\frac{u(m+1, M-m-n-1) P(m+1, n ; k-1)}{u(m+1, M-m-n-1)+u(M-m-n-1, n)} \\
& +\frac{u(M-m-n+1, n-1) P(m, n-1 ; k-1)}{u(m, M-m-n+1)+u(M-m-n+1, n-1)} \\
& =\frac{(m+1)^{\gamma} P(m+1, n ; k-1)}{(m+1)^{\gamma}+n^{\gamma}}+\frac{(n-1)^{\gamma} P(m, n-1 ; k-1)}{m^{\gamma}+(n-1)^{\gamma}},
\end{aligned}
$$

with the initial condition $P(m, n ; 0)=\delta_{m, m_{0}-1} \delta_{n, n_{0}}$. In Fig. 111 we plot $\Delta m$ calculated numerically using the above formula and Eq. (65), for different sizes $m_{0}, n_{0}$ of colliding condensates, and $\gamma=4$. We can see that for $m_{0} \gg n_{0}$, the mass $\Delta m$ transferred in the collision tends to a constant value of about $0.4-0.5$, depending on $\gamma$. In Fig. 12 we show that $\Delta m$ stabilises at 0.5 for $m_{0} \rightarrow \infty$ and $\gamma \rightarrow \infty$. We also see in Fig. 111 that for $m_{0} \approx n_{0}$, the average mass calculated from the recursion relation is actually negative, as if particles flew from the larger to the smaller condensate. This does not agree with Monte-Carlo simulations, see Fig. 8, which indicate that $\Delta m \approx$ const, even for a small difference between $m_{0}, n_{0}$. Since our numerical calculation is exact, this discrepancy implies that in the simulation, clusters collide more than once, until the right cluster becomes sufficiently large compared to the left cluster, and manages to escape.

5.2.3. Coalescence process of clusters Our simulations and numerical calculations thus indicate that we can describe the relaxation towards steady state as a process of coalescence of small clusters into the condensate in a series of collisions. In each 

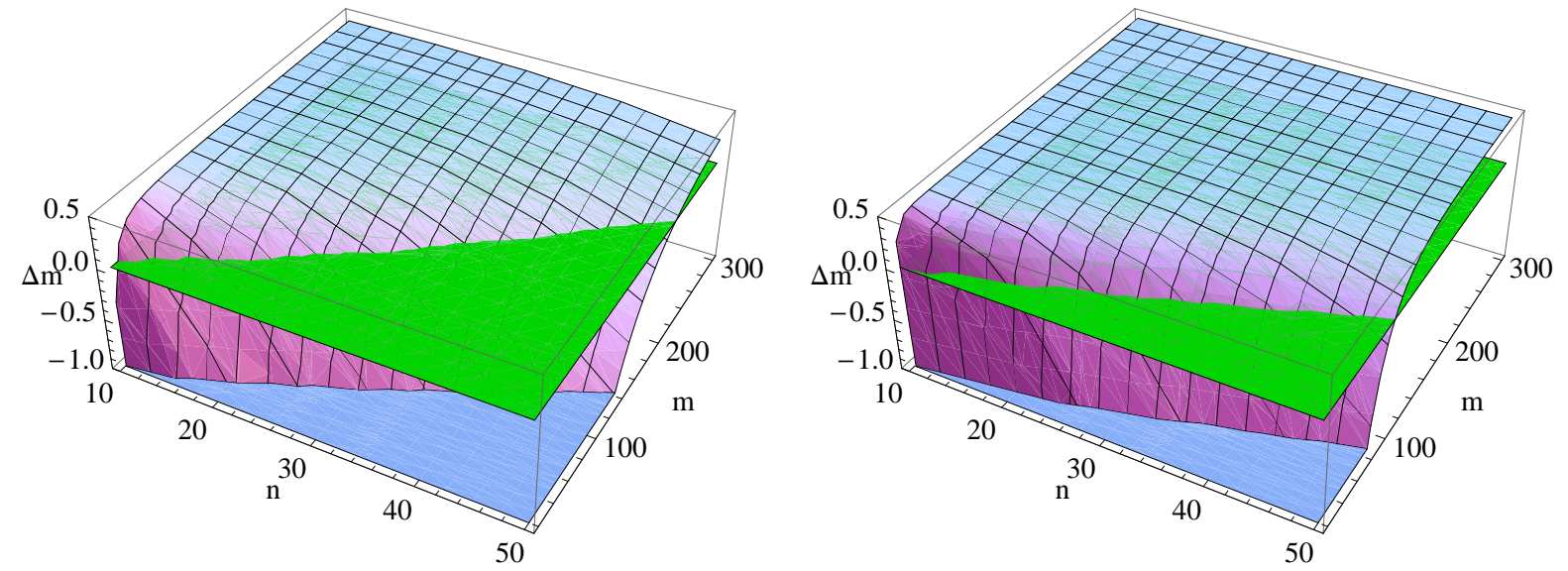

Figure 11. Plots of the average mass $\Delta m$ transferred in a collision of clusters of size $m_{0}, n_{0}$, calculated using recursion (66). Left: $\gamma=4$, right: $\gamma=6$. The half-transparent plane corresponds to $\Delta m=0$. The range of $\Delta m$ has been restricted to $-1, \ldots, 0.5$ for clarity, although for some $m_{0}, n_{0}, \Delta m$ takes values less than -1 .

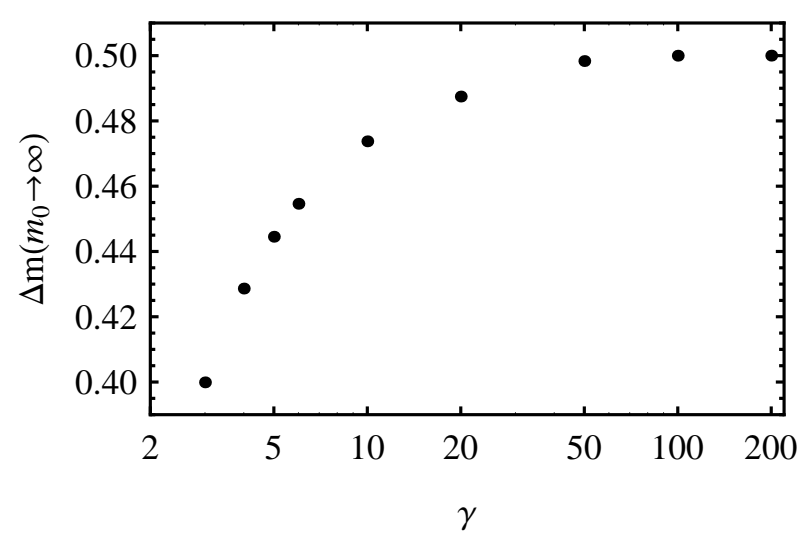

Figure 12. Plot of $\Delta m$ for $m_{0} \rightarrow \infty$ and $n_{0}=10$ as a function of $\gamma$. Transferred mass tends to 0.5 for large $\gamma$.

collision, only a few particles are exchanged on average, and the net flow of particles is from smaller to larger clusters. Let us analyse what happens if the system is initially prepared in a state with $O(L)$ clusters separated by runs of empty sites. Numerically, this can be realised by placing $N=L / W$ clusters at sites $i=1, W, 2 W, \ldots$, where $W$ is some fixed number larger than 1 and independent of $L$. This initial state is not the same as the state with randomly distributed particles that we typically use in simulations, but the existence of well-defined clusters at all times makes the effective description from the previous subsections (in terms of collisions between isolated clusters) applicable to the whole process, from the initial to the final state. Moreover, on a large scale the system still looks homogeneous, as if the particles were distributed uniformly.

Let us first look at the evolution of one cluster. It collides with other clusters, gains or loses particles, and finally it either dissolves into the background or it reaches the steady-state occupation $M-L \rho_{c}$ and becomes the condensate. Let $T$ be the time 


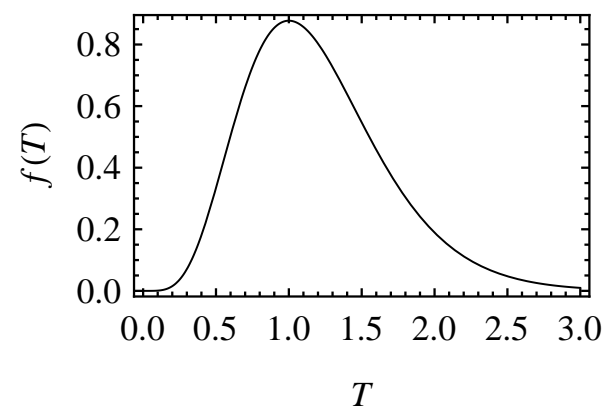

Figure 13. A hypothetical example of the function $f(T)$ which gives the probability that a randomly chosen cluster will become the condensate. The time to steady state is determined by the behaviour of $f(T)$ for $T \rightarrow 0^{+}$.

it takes to reach this steady-state size for this particular condensate $(T=\infty$ if the cluster dissolves into the background). $T$ differs among different clusters and different realisations of the system, and it can be thought of as a random variable with some distribution $f(T)$. In Figure 13 we show a hypothetical form of such a distribution. Since all clusters are initially identical, $f(T)$ is the probability density function of a randomly chosen cluster having evolved into a condensate after time $T$. We have, however, $N$ such clusters and each of them should have the same chance to become the final condensate, although only one of them (the fastest evolving one) will make it. Therefore, the time $T_{\mathrm{ss}}$ to reach steady state will be the minimal time out of times $T_{1}, \ldots, T_{N}$ for individual clusters:

$$
T_{\mathrm{ss}}=\min \left\{T_{1}, \ldots, T_{N}\right\}
$$

and we can estimate it as $T_{\mathrm{ss}}$ such that

$$
\int_{0}^{T_{\mathrm{ss}}} f(T) d T=1 / N
$$

This argument is based on statistical independence of $T_{1}, T_{2}, \ldots, T_{N}$. The rationale behind it is that each cluster can be viewed as moving in a random background formed by other clusters. Since we are interested in large- $N$ (and therefore large- $L$ ) behaviour, we need only the small- $T$ behaviour of the single-cluster distribution $f(T)$.

Let us first, quite generically, write equations for the evolution of mass $m_{n}$ and time $t_{n}$ at which a particular cluster moves by one site to the right and (possibly) exchanges a chunk of mass $\Delta m_{n}$ with other clusters:

$$
\begin{aligned}
& m_{n}=m_{n-1}+\Delta m_{n}, \\
& t_{n}=t_{n-t}+\Delta t_{n} .
\end{aligned}
$$

Here $\Delta m_{n}$ is a random number that can be either zero (the cluster moves to the next site without changing its mass), positive, or negative. We expect that the distribution of $\S$ In Ref. 12] we give a more complicated argument also based on extreme value statistics, which leads however to the same final result. 
$\Delta m_{n}$ should be similar to the distribution $P(\Delta m)$ from Fig. 8, $\Delta t_{n}$ is the time between two jumps and it is exponentially distributed:

$$
p_{n}\left(\Delta t_{n}\right)=\lambda_{n} e^{-\lambda_{n} \Delta t_{n}}
$$

where $\lambda_{n}$ is the rate constant, and we expect that $\lambda_{n} \propto m_{n}^{\gamma}$ for large $m_{n}$. The reason is that for the hop rate (56) and small $\epsilon$ the limiting process is the Poissonian process in which the first particle hops from the condensate to the next, empty site. We now want to calculate the distribution $f(T)$ of the sum $T=t_{1}+t_{2}+\ldots$ since this is the time to reach size $m_{n}=\infty$ (condensation). In Ref. [12] we show that $T$ is a random variable with the following distribution:

$$
f(T) \propto C T^{(1-3 \gamma) /(2(\gamma-1))} \exp \left[-B(A T)^{-\frac{1}{\gamma-1}}\right],
$$

where $B, C$ are some real, positive constants. If we now insert Eq. (72) into Eq. (68) and recall that $N \sim L$, we obtain that the relaxation time asymptotically decreases as

$$
T_{\mathrm{ss}}=c_{1}\left(\ln L+c_{2} \ln \ln L+\ldots\right)^{1-\gamma},
$$

where dots "..." denote terms increasing slower than $\ln \ln L$. For very large $L$, equation (73) predicts that the average time to reach the steady state should decrease as $(\ln L)^{1-\gamma}$. The coefficients $c_{1}, c_{2}$ could be in principle calculated, but since our theory uses many approximations whose errors would accumulate in $c_{1}, c_{2}$, it makes more sense to treat them as free parameters when comparing Eq. (73) with numerical simulations. This is what is done in Fig. 14, where we plot $1 / T_{\mathrm{ss}}$ obtained in simulations, together with Eq. (73) with $c_{1}, c_{2}$ fitted to the data points. The agreement is very good and it extends beyond the assumptions of the above theory. For example, the formula (73) works not only for the initial condition with $N$ isolated clusters of equal size, but also when the clusters have different numbers of particles, or when the initial condition is a random distribution of particles. Moreover, the formula works also when $\epsilon \approx 1$ is not necessarily a small number.

Finally, we note that the effective description does not invoke a factorised steady state or a factorised hop rate. Although our numerical studies presented in Section 5 have used a factorised hopping rate, we checked that similar results are obtained for a choice of dynamics that does not correspond to a factorised hop rate (nor satisfy the conditions for a factorised steady state), but has the same asymptotic behaviour $u(m, n) \sim(m n)^{\gamma}$.

\section{Explosive condensation as a spatially-extended example of instantaneous gelation.}

As we have seen an effective description of explosive condensation which occurs for $v(m) \sim m^{\gamma}$ is given in terms of coalescence of clusters through collisions. In turn this description bears some resemblance to a coagulation process. These processes describe aggregation of clusters of particles that can be anything from atoms to stars, and have applications to such diverse problems as gravitational clustering [33], formation 

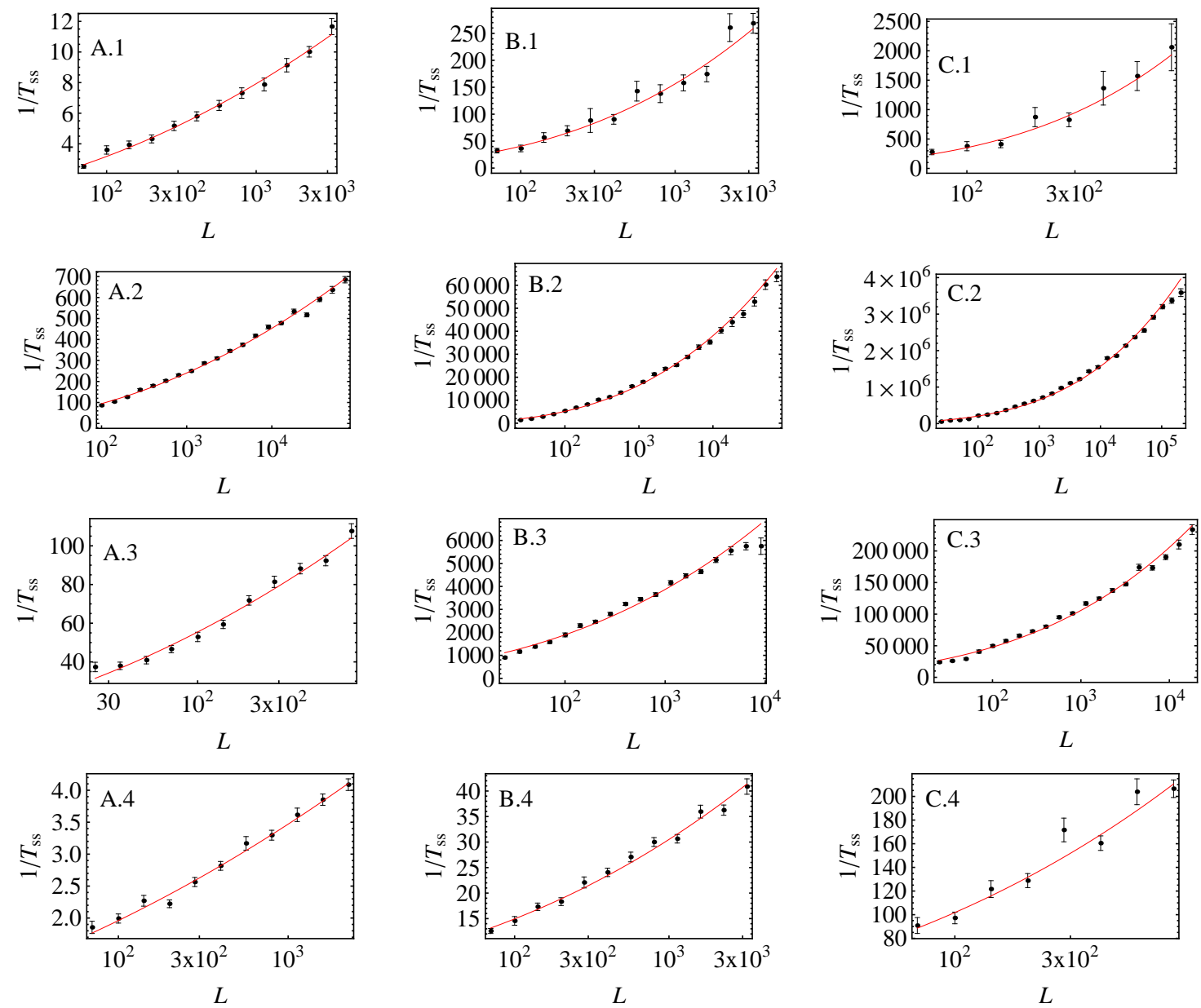

Figure 14. Plots of $T_{\mathrm{ss}}^{-1}$ as a function of $L$ (points). Solid line is Eq. (73) fitted to the data points. Columns A-C: $\gamma=3,4,5$, from left to right. Rows 1-4 correspond to 1) Poissonian initial distribution of particles, $v(m)=(m+0.3)^{\gamma}$, 2) Poissonian distribution, $v(m)=(m+1)^{\gamma}$, 3) Poissonian distribution with only every 5th site occupied, $\left.v(m)=(m+1)^{\gamma}, 4\right)$ the same but $v(m)=(m+0.3)^{\gamma}$.

of droplets in clouds due to collisions [34], and differential sedimentation [35]. What all these processes have in common is that the rate at which clusters merge and form larger clusters increases with their size. A particularly simple example is the growth of raindrops that fall through the mist under their own gravity. Since the terminal velocity of a raindrop increases with its size, and the rate of mass accretion will be proportional to the volume "swept" by the drop, the drop will aggregate mass at an increasing rate as it grows [36]. In essence this is the common feature with explosive condensation described in the previous section.

The process of irreversible cluster-cluster aggregation is usually described by Smoluchowski equation [37]:

$$
\frac{d N_{i}}{d t}=\frac{1}{2} \sum_{j+k=i} K(j, k) N_{j} N_{k}-\sum_{j} K(i, j) N_{i} N_{j} .
$$

Here $N_{i}$ is the number of clusters of size $i$. The function $K(i, j)$, which gives the rate 
at which clusters of sizes $i$ and $j$ merge, is called the coagulation kernel. In theoretical studies of aggregation processes, it is often assumed that the kernel is a homogeneous function,

$$
K(a i, a j)=a^{\lambda} K(i, j),
$$

where $\lambda$ is the degree of homogeneity. Many kernels that arise from practical applications are homogeneous, so this restriction is very mild. One form for a kernel satisfying (75) is

$$
K(i, j)=i^{\nu} j^{\mu}+i^{\mu} j^{\nu}
$$

with $\nu>\mu$ and the degree of homogeneity $\lambda=\mu+\nu$. Although in the limit of infinitely large systems the final state - a single, infinite cluster - is the same regardless of $\nu, \mu$, the dynamics of this "gelation" process (where the infinite cluster plays the role of gel) depends on $\lambda$ and $\nu$. For $\lambda<1$, the size of the largest cluster (gel) grows as a power of time, and gelation takes infinitely long. For $\lambda=1$, gelation still takes infinite time but the size of the gel grows exponentially. For $\lambda>1$, however, gelation takes finite time for $\nu<1$, or is believed to happen in zero time $\nu>1$ [37, 38, 39]. This latter process is called "instantaneous gelation".

Instantaneous gelation bears a close resemblance to our explosive condensation, with time $T_{\mathrm{ss}}$ to steady state decreasing as $(\ln L)^{1-\gamma}$ and hence becoming zero for $L \rightarrow \infty$. However, there are also important differences between our model and instantaneous gelation. First, clusters can split apart in our model, hence aggregation is reversible (albeit this occurs rarely for large clusters). The process of merging of clusters is also different to that of the Smoluchowski equation; our clusters do not completely merge upon contact but only exchange particles, with a net flow of particles from smaller to larger cluster. This is not a serious difference; we could imagine rescaling the kernel so that the aggregation rate would correspond to the same effective net flow. However, since in our model the rate of collisions is proportional to the relative velocity of clusters $i, j$, the effective exchange kernel would have to assume the following form:

$$
K(i, j) \sim i^{\gamma}-j^{\gamma}
$$

for $i>j$, and $K(i, j)=0$ otherwise. This is quite different to Eq. (76) which is symmetric in $i, j$. However, in the limit $i \gg j$, which is relevant when one of the clusters begins to dominate, kernel (177) reduces to Eq. (76) with $\nu=\gamma$. Interestingly, in this regime our model becomes similar to exchange driven growth [40] in which particles are transferred between clusters of size $i$ and $j$ at a rate $K(i, j)$ given by Eq. (76). The model [40] leads to instantaneous gelation for $\nu>2$, which corresponds to $\gamma>2$ in our model. However, the fact that our effective kernel (77) is not symmetric in $i, j$ makes a difference in the scaling of the time to steady state. Specifically, our model predicts $T_{\mathrm{ss}} \sim(\ln L)^{-(\gamma-1)}$, whereas exchange driven growth [40] gives $T_{\mathrm{ss}} \sim(\ln L)^{-(\gamma-2)}$. Finally we should bear in mind that the explosive condensation occurs on a spatially extended one-dimensional lattice whereas coagulations processes are essentially mean field models defined without any dimensionality 


\section{Conclusion}

In this work we have considered the factorised steady states in the misanthrope process and the condensation phenomena that may occur. We have seen that for the standard condensation scenario (case I of section 4) there are distinct type of dynamics that will yield the same required asymptotic behaviour of the single-site weight $f(m)$. This is in contrast to the ZRP. We have also seen how the existence of condensation may depend not only on the asymptotic behaviour of the hop rates but also on the behaviour of the hop rate for small $m$; specifically, condensation may or may not be possible, depending on the value $v(0)$ of the function $v(m)$ introduced in section 3.1.

This result may be of significance for example to the phase separation criterion proposed for driven diffusive systems [41, 42]. There it was assumed that one could model domains in the driven system as sites in a zero range process and relate the current out of a domain as the hopping rate of the zero range process (depending only on the domain size). Phase separation in the driven system then corresponds to condensation in the Zero range process. If the current between two neighbouring domains has a more complicated relationship between the two domain sizes, a description in terms of a misanthrope process might become more appropriate.

We have also analysed the dynamics of condensation and showed that if $v(m)$ increases as a power of $m$ (cf. equation (38)), the time to steady state decreases with increasing system size $L$. This means that in the limit $L \rightarrow \infty$, the steady-state condensate is produced instantaneously. This is at variance with ZRP-like dynamics (39) and resembles instantaneous gelation known from the theory of coagulation processes discussed in Sec. 6. However, in contrast to these models with mean-field dynamics, our misanthrope process provides a non-trivial example of instantaneous gelation in a spatially-extended system. Since our analysis is quite generic and is not based on the factorization of the steady state, we expect that the same dynamics will hold in any non-equilibrium system with hop rates increasing with occupation numbers.

Although our analysis of the dynamics is well confirmed by numerical simulations, it remains to be seen if a seemingly simple expression (173) for the time to condensation $T_{\mathrm{ss}}$ can be derived without various approximations that we have assumed. In particular, it would be interesting to see whether the same scaling $T_{\mathrm{ss}} \sim(\ln L)^{1-\gamma}$ remains true for an arbitrary initial condition.

\section{Acknowledgments}

We would like to thank D. Mukamel, S. Grosskinsky, and C. Connaughton for helpful discussions. B.W. was supported by a Leverhulme Trust Early Career Fellowship. This work was funded in part by the EPSRC under grant number EP/J007404/1, 


\section{Appendix A}

We shall show that the most general form of a function $F(a, b)$ which obeys the equation

$$
\sum_{i=1}^{L} F\left(m_{i}, m_{i+1}\right)=0,
$$

with $m_{L+1} \equiv m_{1}$, which holds for any numbers $m_{1}, \ldots, m_{L}$ with $L>2$, is $F(a, b)=$ $h(a)-h(b)$. The first step is to take derivatives with respect to $m_{j}, m_{j+1}$, which leads us to

$$
\frac{\partial^{2} F\left(m_{j}, m_{j+1}\right)}{\partial m_{j} \partial m_{j+1}}=0 .
$$

Integrating the above equation over $m_{j}, m_{j+1}$ we obtain that $F(m, n)$ which obeys (79)), can be most generally expressed as

$$
F(m, n)=h_{1}(m)+h_{2}(n),
$$

with some arbitrary functions $h_{1}(m), h_{2}(n)$. The form (80) is only a necessary condition to have Eq. (78) fulfilled. To find the sufficient condition, we insert (80) into (78) and obtain

$$
\sum_{i}\left[h_{1}\left(m_{i}\right)+h_{2}\left(m_{i}\right)\right]=0 .
$$

Varying one of $m_{i}$ 's while keeping other variables fixed we see that it must be $h_{1}(m)=-h_{2}(m)$. Defining now $h(m) \equiv h_{1}(m)$ we see that $F(m, n)=h(m)-h(n)$ which concludes our proof.

Interestingly, one can also show in a similar way that if $F\left(a_{1}, a_{2}, \ldots, a_{N}\right)$ depends on $N>2$ variables, then the most general solution to the equation

$$
\sum_{i=1}^{L} F\left(m_{i}, m_{i+1}, \ldots, m_{i+N-1}\right)=0
$$

is $F\left(a_{1}, a_{2}, \ldots, a_{N}\right)=h\left(a_{1}, a_{2}, \ldots, a_{N-1}\right)-h\left(a_{2}, a_{3}, \ldots, a_{N}\right)$, provided that we demand the solution to be the same, irrespective of the number of sites $L>N$.

\section{Appendix B}

We shall show that the condition (13)

$$
u(m, n)=u(m+1, n-1) \frac{u(1, m) u(n, 0)}{u(m+1,0) u(1, n-1)}+u(m, 0)-u(n, 0)
$$

on the factorization of the steady state in the misanthrope process reduces in fact to two equations (14) and (15):

$$
\begin{aligned}
& u(n, m)=u(m+1, n-1) \frac{u(1, m) u(n, 0)}{u(m+1,0) u(1, n-1)}, \\
& u(n, m)-u(m, n)=u(n, 0)-u(m, 0) .
\end{aligned}
$$


We will prove Eqs. (84) and (85) by induction. Let us first replace $n$ by $n=m+p$ in Eq. (85), which gives

$$
u(m+p, m)-u(m, m+p)=u(m+p, 0)-u(m, 0) .
$$

It follows then from Eq. (83) that

$$
\begin{aligned}
u(m, m+p)= & u(m+1, m+p-1) \frac{u(1, m) u(m+p, 0)}{u(m+1,0) u(1, m+p-1)} \\
& +u(m, 0)-u(m+p, 0) .
\end{aligned}
$$

Using Eq. (86) and changing variables $m \rightarrow m-1, p \rightarrow p+2$ we obtain

$$
u(m, m+p)=u(m+p+1, m-1) \frac{u(1, m+p) u(m, 0)}{u(m+p+1,0) u(1, m-1)} .
$$

This equation stems from (yet-to-be proved) Eqs. (86) and known Eq. (83). It is therefore enough to prove only Eq. (86) by induction. Equation (866) is clearly fulfilled for $p=0$. For $p=1$, which corresponds to $n=m+1$, we have from Eq. (83) that

$$
\begin{aligned}
u(m, m+1) & =u(m+1, m) \frac{u(1, m) u(m+1,0)}{u(m+1,0) u(1, m)}+u(m, 0)-u(m+1,0) \\
& =u(m+1, m)+u(m, 0)-u(m+1,0),
\end{aligned}
$$

which is equivalent to Eq. (86) with $p=1$. We thus know that Eq. (86) holds for $p=0,1$. To prove it for arbitrary $p$, we again use Eq. (83) with $m \rightarrow m-1, n \rightarrow m+p+1$ :

$$
\begin{aligned}
u(m-1, m+p+1) & =u(m, m+p) \frac{u(1, m-1) u(m+p+1,0)}{u(m, 0) u(1, m+p)} \\
& +u(m-1,0)-u(m+p+1,0),
\end{aligned}
$$

and by inserting $u(m, m+p)$ from Eq. (88) we obtain

$$
u(m-1, m+p+1)=u(m+p+1, m-1)+u(m-1,0)-u(m+p+1,0),
$$

which is equivalent to Eq. (86) but with $m, p$ replaced by $m-1$ and $p+2$, respectively. We have thus proved that equations (86) and (88) are fulfilled for any $p$ which completes our proof.

\section{Appendix C}

We will prove that the Misanthrope process cannot be extended to a pair-factorized steady state by relaxing the constraint (13) (or, equivalently, Eqs. (14)15)) on the hop rate. We start by supposing that this is not the case and one can find $g(m, n)$ such that the equation for the probability current (6) is fulfilled by

$$
P\left(m_{1}, \ldots, m_{L}\right)=\prod_{i=1}^{L} g\left(m_{i}, m_{i+1}\right) .
$$

Following the same lines as in Sec. 2 and using results of Appendix A for $N=3$, we arrive at a relation between $u(m, n), g(m, n)$ and an auxiliary function $h(m, n, k)$ :

$$
\begin{aligned}
& u(l, m)-u(l+1, m-1) \frac{g(k, l+1) g(l+1, m-1) g(m-1, n)}{g(k, l) g(l, m) g(m, n)} \\
& =h(k, l, m)-h(l, m, n) .
\end{aligned}
$$


Our first task is to express $h(k, l, m)$ through the hop rate. We will do this in three steps. First, by assuming $m=0$ we have from Eq. (93) that

$$
u(l, 0)=h(k, l, 0)-h(l, 0, n) .
$$

The left-hand site depends on $l$ only and hence it must be that $h(k, l, 0)$ and $h(l, 0, n)$ depend only on their second and first arguments, respectively. Assuming $l=0$ in Eq. (93) we obtain that

$$
0=u(1, m-1) \frac{g(k, 1) g(1, m-1) g(m-1, n)}{g(k, 0) g(0, m) g(m, n)}+h(k, 0, m)-h(0, m, n) .
$$

However, we have just shown that $h(k, 0, m)$ is a function of $k$ only. The above equation thus depends on $k$ only through $h(k, 0, m)$ and $g(k, 1) / g(k, 0)$, the last term being multiplied by the function of $m, n$. The equation has to be valid for any $m, n, k$, and hence it must be $h(k, 0, m) \equiv H=$ const and $g(k, 1) / g(k, 0) \equiv G=$ const. We can now calculate the ratio $g(m-1, n) / g(m, n)$ from Eq. (95):

$$
\frac{g(m-1, n)}{g(m, n)}=G \frac{[h(0, m, n)-H] g(0, m)}{g(1, m-1) u(1, m-1)} .
$$

Assuming now $n=0$ and inserting Eq. (96) into Eq. (93) we obtain

$$
\begin{aligned}
& u(l, m)-u(l+1, m-1) \frac{g(k, l+1) g(l+1, m-1)}{g(k, l) g(l, m)} \\
& \times G \frac{h(0, m, 0)-H}{u(1, m-1)} \frac{g(0, m)}{g(1, m-1)} \\
& =h(k, l, m)-h(l, m, 0) .
\end{aligned}
$$

From Eq. (94) we obtain that $h(l, m, 0)=u(m, 0)+H$ which leads to the expression for $h(k, l, m)$ :

$$
\begin{aligned}
& h(k, l, m)=u(m, 0)+H+u(l, m) \\
& -u(l+1, m-1) G \frac{g(k, l+1) g(l+1, m-1) g(0, m) u(m, 0)}{g(k, l) g(l, m) g(1, m-1) u(1, m-1)} .
\end{aligned}
$$

Next, we insert $h(k, l, m)$ from Eq. (98) into the initial equation (93) and, after some calculations, we obtain

$$
\begin{aligned}
& \frac{g(k, l+1)}{g(k, l)} u(l+1, m-1) \frac{g(l+1, m-1) G g(0, m)}{g(l, m) u(1, m-1) g(1, m-1)}[u(n, 0)+u(m, n) \\
& \left.-u(m+1, n-1) \frac{g(0, m+1) g(m+1, n-1) g(0, n) G u(n, 0)}{g(0, m) g(m, n) g(1, n-1) u(1, n-1)}-u(m, 0)\right] \\
& =[u(n, 0)+u(m, n)-u(m, 0) \\
& \left.-u(m+1, n-1) \frac{g(l, m+1) g(m+1, n-1) g(0, n) G u(n, 0)}{g(l, m) g(m, n) g(1, n-1) u(1, n-1)}\right] .
\end{aligned}
$$

The only dependence on $k$ in the above equation is through the ratio $g(k, l+1) / g(k, l)$. To be valid for all $k$, this ratio has to be $k$-independent or the expressions in square brackets must vanish. In the first case, the only possibility is that $g(k, l)$ factorizes into a product of two functions of separately $k$ and $l$. In the second case, the only dependence 
on $l$ in the right-hand side square bracket is through $g(l, m+1) / g(l, m)$. Using the same reasoning we again obtain the factorization of $g(l, m)$. Inserting a factorized form, e.g.,

$g(m, n)=\sqrt{f(m) f(n)}$ into the above equation, one arrives at the constraint (13) for the fully factorized misanthrope process. We thus see that the extension of the misanthrope process to pair-factorized steady states by relaxing the constraint (13) is impossible.

\section{References}

[1] Burda Z, Johnston D, Jurkiewicz J, Kaminski M, Nowak M A, Papp G, and Zahed I, 2002 Phys. Rev. E 65, 026102.

[2] Bialas P, Burda Z, Petersson B and Tabaczek J, 1997 Nucl. Phys. B 495, 463.

[3] Evans M R, Majumdar S N, Pagonabarraga I, and Trizac E, 2010 J. Chem. Phys. 132, 014102.

[4] Krapivsky P L, Redner S, and Leyvraz F, 2000, Phys. Rev. Lett. 85, 4629.

[5] O'Loan O J, Evans M R and Cates M E, 1998 Phys. Rev. E 58, 1404.

[6] Chowdhury D, Santen L, Schadschneider A, 2000 Physics Reports, 329, 199-329.

[7] Spitzer F, 1970 Adv. Math. 5246.

[8] Kelly F P, Reversibility and Stochastic Networks, Wiley (1979).

[9] Cocozza-Thivent C, 1985 Z. Wahr. Verw. Gebiete 70 509-523.

[10] Evans M R and Hanney T, 2005 J. Phys. A: Math. Gen. 38, R195.

[11] Luck J-M and Godrèche C, 2007 J. Stat. Mech.: Theor. Exp. P08005.

[12] Waclaw B and Evans M R, 2012, Phys. Rev. Lett. 108, 070601; see also Supplemental Material therein.

[13] Chui S T, Weeks J D 1981 Phys. Rev. B, 232438.

[14] Burkhardt T W 1981 J. Phys. A: Math. Gen., 14 L63.

[15] van Leeuwen J M L, Hilhorst H J 1981 Physica A, 107319.

[16] Evans M R, Hanney T, and Majumdar S N, 2006 Phys. Rev. Lett. 97, 010602.

[17] Ferrari P A Private Communication

[18] Godrèche C, 2007 Lect. Notes Phys. 716, 261.

[19] Waclaw B, Sopik J, Janke W, and H. Meyer-Ortmanns, 2009 Phys. Rev. Lett. 103, 080602.

[20] Schütz G M and Sandow S, 1994 Phys. Rev. E 49, 2726.

[21] Grosskinsky S, Redig F and Vafayi K, 2011 J. Stat. Phys. 142, 952.

[22] Gabel A, Krapivsky P L, Redner S, 2010 Phys. Rev. Lett. 105, 210603.

[23] Bialas P, Burda Z, Johnston D, 1997 Nuclear Physics B 493, 505.

[24] Evans M R, Majumdar S N, and Zia R K P, 2006 J. Stat. Phys 123, 357-390.

[25] Ritort F, 1995 Phys. Rev. Lett. 75, 1190-1193

[26] Franz S and Ritort F, 1997 J. Phys. A: Math. Gen. 30, L359-L365.

[27] Andrews G E, Askey R, Roy R Special Functions, Chapter 2, (Cambridge University Press, 1999).

[28] Godrèche C, 2003 J. Phys. A: Math. Gen. 36, 6313.

[29] Godrèche C and Luck J-M, 2005 J. Phys. A: Math. Gen 38, 7215-7237.

[30] Hirschberg O, Mukamel D and Schutz G M, 2009 Phys. Rev. Lett. 103, 090602.

[31] Hirschberg O, Mukamel D, and Schütz G M. 2012 J. Stat. Mech.: Theor. Exp. P08014.

[32] Hirschberg O, Mukamel D, and Schütz G M, 2013 Phys. Rev. E 87, 052116.

[33] Silk J and White S D, 1978 Astrophys. J. 223, L59.

[34] Falkovich G, Fouxon A, and Stepanov M G, 2002 Nature 419, 151.

[35] Horvai P, Nazarenko S V, and Stein T H M, 2008 J. Stat. Phys. 130, 1177.

[36] Edwards B F, Wilder J W, and Scime E E, 2001 Euro J. Phys. 22, 113.

[37] Connaughton, Colm, Rajesh, R. and Zaboronski, Oleg V. (2011) Kinetics of cluster-cluster aggregation. In: Sattler, Klaus D., (ed.) Handbook of nanophysics, CRC Press, pp. 1-40.

[38] van Dongen P G J, 1987 J. Phys. A: Math. Gen. 201889.

[39] Leyvraz F, 2012 J. Phys. A: Math. Theor. 45, 125002. 
[40] Ben-Naim E and Krapivsky P L, 2003 Phys. Rev. E 68031104.

[41] Kafri Y, Levine E, Mukamel D, Schütz G M and Török J, 2002 Phys. Rev. Lett. 89, 035702.

[42] Evans M R, Levine E, Mohanty P K, Mukamel D, 2004 Process Eur. Phys. J. B 41, 223-230. 